Saudi Journal of Biomedical Research

Abbreviated Key Title: Saudi J Biomed Res ISSN 2518-3214 (Print) |ISSN 2518-3222 (Online) Scholars Middle East Publishers, Dubai, United Arab Emirates Journal homepage: https://saudijournals.com

Review Article

\title{
Intersectins as Novel Protein for Viral Endocytosis- A Mini Review
}

Dluya Samuel Thagriki ${ }^{1,2,3^{*}}$

${ }^{1}$ CSIR-Indian Institute of Chemical Biology, 4, Raja S.C., Mullick Road, Jadavpur, Kolkata-700032, West Bengal, India

${ }^{2}$ Academy of Scientific and Innovative Research (AcSIR), Ghaziabad- 201002, India

${ }^{3}$ Department of Biochemistry, Adamawa State University Mubi, Adamawa State, Nigeria-West Africa

DOI: $10.36348 /$ sjbr.2022.v07i01.003

| Received: 16.12.2021 | Accepted: 21.01.2022 | Published: 24.01.2022

*Corresponding author: Dluya Samuel Thagriki

Academy of Scientific and Innovative Research (AcSIR), Ghaziabad- 201002, India

\section{Abstract}

Intersectins (INSTNs) have been defined as scaffold proteins that have several biochemical functions. Initially INSTNs are described as endocytic regulators and function in different signalling pathways including Ras and Ras-like GTPases, PI3Ks and RTKs in several diseases. Recently, INSTNs have been reported in viral endocytosis. INSTNs was found to be upregulated in transcriptomic analysis of differentially expressed protein from Dengue virus serotype four from our recent data. Hence, this review summarises the role of INSTNs in viral endocytosis by interaction with signalling pathways. INSTN-2 interact with K15 protein of Kaposi's SarcomaAssociated Herpesvirus by selective SH3 domain, INSTN-1 interaction with Epstein-Barr virus Latent Membrane Protein 2 (LMP2A), while direct interaction of NPF motifs at C-terminus of A36 with INSTN-1 and Epsin15 homology domains recruiting AP-2 and clathrin mediate viral release was reported in Vaccinia virus. Most recent, INSTN-2 was shown to regulate adaptive immune response during viral infectivity. This was discovered when mice deficient in INSTN-2 exhibited high mortality rate, impaired antibody responses to vaccination and reduced germinal centre formation in Influenza A virus. This review revealed INSTNs as essential viral endocytic proteins. The review also highlights INSTNs role in other diseases and the regulation of signalling pathways. Targeting INSTNs could be important for the development of pharmaceuticals against viruses and diseases. More study is required to establish the mechanistic role of INSTNs for viral endocytosis.

Keywords: Intersectins, signalling pathway, endocytosis, virus, regulation.

\section{Graphical Abstract}

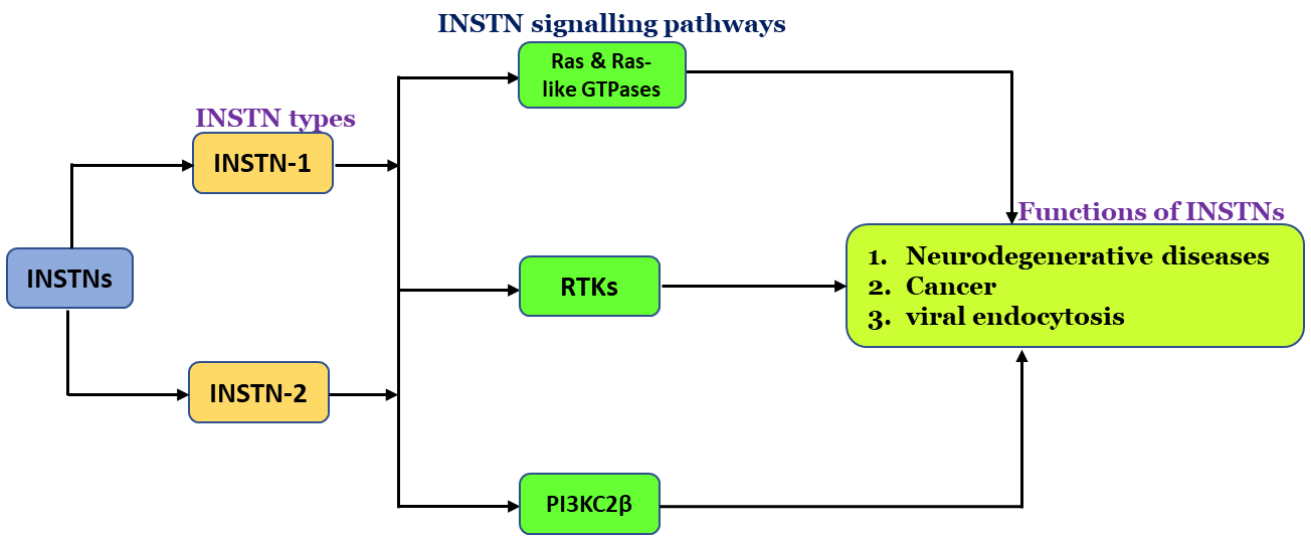

$\begin{array}{ll}\text { List of Abbreviations } \\ \text { INSTN: } & \text { Intersectin } \\ \text { EH: } & \text { Eps15 homology } \\ \text { SH3: } & \text { Src homology 3-domain } \\ \text { DH: } & \text { Dbl homology } \\ \text { PH: } & \text { Pleckstrin homology } \\ \text { CCP: } & \text { Clathrin-coated pit } \\ \text { GEF: } & \text { G-nucleotide exchange factor (GEF) } \\ \text { Ras: } & \text { Rat sarcoma virus } \\ \text { Sos: } & \text { Salt Overly Sensitive } \\ \text { EGF: } & \text { Epidermal growth factor } \\ \text { ERK: } & \text { Extracellular signal-regulated kinase } \\ \text { EGFR: } & \text { Epidermal growth factor receptor }\end{array}$

Citation: Dluya Samuel Thagriki (2022). Intersectins as Novel Protein for Viral Endocytosis-A Mini Review. Saudi J 
JNK:

PI3K:

PI3KC2 8 :

GAP:

PDGF:

Cdc42:

RBD:

GTP:

Ephb2:

RTKs:

AP2:

WASp:

WIP:

LMP2A:

NPFs:

MMP:

DS:

AD:

MAPK:
c-Jun amino-terminal kinase

Phosphatidylinositol 3-kinase

Phosphatidylinositol 3-kinase Class 2beta

GTPase activating protein

Platelet-derived growth factor

Cell division control protein 42 homolog

Ras binding domain

Guanosine triphosphate

EphrinB2

Receptor tyrosine kinases

Adaptor protein 2

Wiskott-Aldrich syndrome (WASp)

WASP-interacting protein

Latent Membrane Protein 2

Nucleation promoting factors (NPFs)

Matrix metalloproteinase

Down Syndrome

Alzheimer Disease

Mitogen-activated protein kinase.

Copyright () 2022 The Author(s): This is an open-access article distributed under the terms of the Creative Commons Attribution 4.0 International License (CC BY-NC 4.0) which permits unrestricted use, distribution, and reproduction in any medium for non-commercial use provided the original author and source are credited.

\section{INTRODUCTION}

Intersectins (INSTN) are scaffold proteins having exceptional multidomain structure. INSTNs binds to several proteins. Their multimeric complexes are implicated in clathrin and caveolin mediated endocytosis, actin cytoskeleton, and cell signalling [15]. INSTNs was first detected in Xenopus [6, 7]. The genes encode two key protein isoforms arising from differential splicing. Structural presentation of INSTNs is shown in Figure 1. INSTN short isoform (INSTN-S) has two N-terminal Eps15 homology (EH1 and EH2) domains having a coil-coiled (CC) region along with five Src homology 3 (SH3A-SH3E) domains. The short isoforms (INSTN-S) are expressed ubiquitously [8-12]. Additionally, INSTN has a long isoform (INSTN-L), it is jointed with INSTN-S. INSTN-L has an extended Cterminal encoding a Dbl homology (DH) domain, a pleckstrin homology (PH) domain, and a $\mathrm{C} 2$ domain. Two isoforms of INSTN-L were found in mammals (INSTN1-L and INSTN2-L). INSTN1-L was specifically expressed in the brain while INSTN2-L was expressed ubiquitously [13]. INSTNs was reported to have role in endocytosis due to the existence of $\mathrm{EH}$ domains. Several studies have also denoted the direct interaction of the EH domain with certain components of the endocytic machinery including Epsin-1, Epsin-2, SCAMP1, Stonin2 and Rev interacting protein (Rip/RAB/Hrb). [7, 14-16]. Furthermore, INSTN was reported to regulate clathrin-dependent endocytosis, play conserved role in endocytosis and also found to localizes clathrin-coated vesicles [12, 17-21]. INSTNs has been reported to be important components for the formation of clathrin-coated pit (CCP) [22]. Studies have shown that INSTNs act as mediators of Ras/MAPK signalling pathway [5, 23]. Despite the significant role of INSTNs in endocytosis, little is still known on the INSTNs function in viral endocytosis. Hence, this review discussed the recent findings on INSTNs role in viral endocytosis, their interacting partners and different signalling pathways utilized by INSTNs that enhanced viral infectivity.

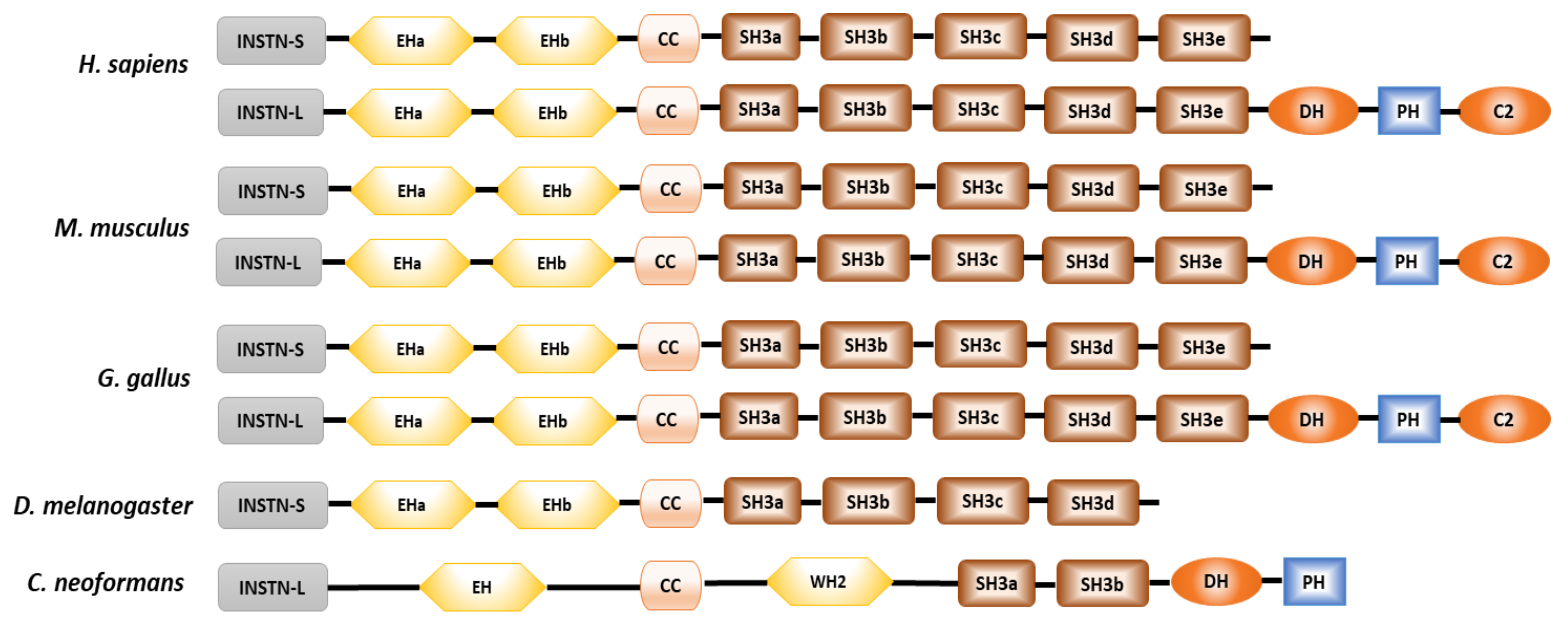

Figure 1: Structure of Intersectin (INSTN). INSTN consist of Eps 15 homology domains tagged EH, a coil-coil domain tagged CC and multiple Src homology 3 domain tagged SH3. Each domain has distinct ligands. Many organisms have longer splice product labelled INSTN-L which shares the domains with a shorter splice product labelled INSTN-S. Additionally, it has a C-terminus encoding Rho exchange factor domain (DH) that activate Cdc42 in connection with the PH domain. INSTN ortholog revealed nearly all INSTNs have two EH domains and five SH3 domains. Lower organisms like $C$. neoformans INSTN Cin1 has only one EH domain and two SH3 domains but no C2 domain in the longer INSTN-L. Additionally, the Cin1 has an attached WH2 domain which bind actin 
Dluya Samuel Thagriki., Saudi J Biomed Res, Jan, 2022; 7(1): 25-32

\section{INSTN signalling pathways}

Up till now INSTNs uses mainly three signalling pathways for regulating its activities. Here, this review therefore discussed in brief these signalling pathways.

\subsection{Regulation of Ras and Ras-like GTPases}

O'Bryan in one of his famous articles depicted INSTNs regulating Ras and Ras-like GTPases [21]. Tong and colleagues reported that INSTN-1 SH3 domains bind Ras, GEF and Sos proline rich regions while overexpression of INSTN SH3 domain result in blockade of growth factor-mediated Ras activation [23, 24]. Contrary, Mohney and group reported INSTN-1S overexpression activate $\mathrm{H}$-Ras on perinuclear vesicles [3]. Furthermore, overexpression of $\mathrm{SH} 3$ domains of INSTN-1 hinders EGF activation of Ras and ERK [23]. This may signify INSTN-1 activating ERK pathway, although overexpression of INSTN-1S does not stimulate ERK pathway, [17] alternatively INSTN-1S indirectly affects ERK activation through stimulating EGFR internalization [25] and results in the activation of ERK by EGFR [26-27]. Despite the fact that INSTN1 interaction with the Ras family members has not been completely discovered, however, interaction of Sos therefore indicate that INSTN-1 may be regulating NRas and K-Ras. Mor and Philips [28] reported H-Ras and N-Ras function in both plasma membrane and intracellular compartment while K-Ras was recently reported for its role in intracellular vesicles and plasma membrane [29]. It will be important if INSTNs function can be further investigated in all Ras isoforms. Although the importance of INSTN-H-Ras is still undefine, INSTN-1-H-Ras does not activate ERK or JNK pathways [3]. This may also mean that INSTN-1$\mathrm{H}$-Ras stimulates different signalling pathway. Activation of PI3K class $2 \beta$ (PI3K-C2 $\beta$ ) by INSTNs on vesicles [4], interaction of INSTN with Ras and PI3K-
$\mathrm{C} 2 \beta$ and the appearance of Ras binding domain in PI3K-C2 $\beta$, showed that INSTN-1-H-Ras regulate the activation of PI3K-C2 $\beta$ on vesicles [3]. The Racspecific DH-PH module on Sos results in additional possibility that INSTN-Sos complex may similarly regulate Rac activation [30]. INSTN-1 may indirectly regulate Rac activation via the hampering CdGAP, Rac and Cdc42-specific GTPase activating protein (GAP) [31]. Expression of INSTN hampered CdGAP activity in vitro and in vivo resulting in heightening lamellipodia formation following platelet-derived growth factor (PDGF) stimulation. Hence, INSTN-1 may regulate Cdc42 through activation and increased exchange of GTP (GEFs) or by preventing its inactivation through hampering of GTPase activity. It could be deduced therefore that INSTN may be a link for the regulation of multiple Ras-like GTPases [32].

\subsection{Regulation of receptor tyrosine kinases}

Studies have shown that INSTN interacts with EphB2 for regulation of dendritic spine morphogenesis via activation of $\mathrm{Cdc} 42$ and modulation of the actin cytoskeleton [33, 34]. Furthermore, INSTN combines with EGFR and trigger gene expression via JNK MAPK pathway $[3,17]$. Since INSTN does not directly activate ERK, INSTN knockdown decreases ERK activation and EGF stimulation $[3,17,25]$, leading to decrease in EGFR internalization [25, 27]. INSTN similarly reduces RTK activity through enhancing ubiquitylation of EGFR by ubiquitin E3 ligase $\mathrm{Cbl}$ due to growth factor stimulation [25]. Even though the INSTN Cbl regulatory mechanism is still unknown, INSTN however showed no alteration in the phosphorylation position of $\mathrm{Cbl}$ and its relation with EGFR. Since $\mathrm{Cbl}$ regulation is a complex process [35]. INSTN may boost its relation with $\mathrm{Cbl}$ and activators or block $\mathrm{Cbl}$ interaction with inhibitors [25]. Regulation of RTKs by INSTN is summarized in Figure 2.

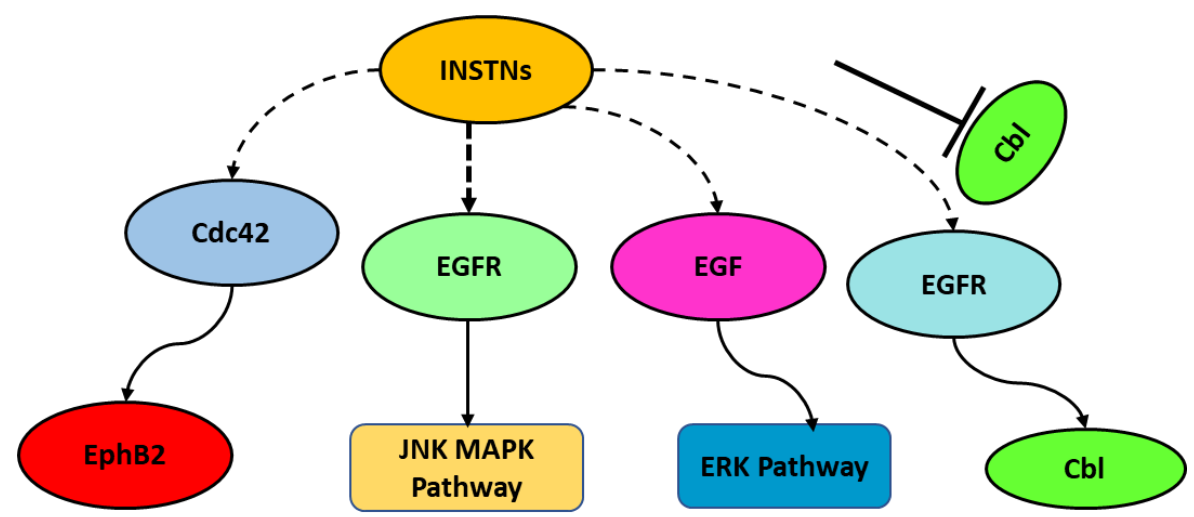

Figure 2: Regulation of receptor tyrosine kinases. INSTNs interact with EphB2 through activation of Cdc42. ITSNs interact with EGFR and stimulate gene expression via the JNK MAPK pathway. INSTNs also interact with ERK pathway through EGF stimulation. INSTN enhancing $\mathrm{Cbl}$ association with activators or block interaction of $\mathrm{Cbl}$ with inhibitors

\subsection{Regulation of phosphatidylinositol 3-kinase Class 2beta (PI3KC2 $\beta)$ \\ PI3Ks are family of conserved lipid kinases} known to phosphorylate 3' position of phosphatidylinositol. PI3Ks belonging to class II which include PI3K-C2 $\beta$. It comprises of a single 175-200 $\mathrm{kDa}$ catalytic subunits that mostly associates with endocytic vesicles and are deficient in adaptor regulatory subunit [36-38]. PI3Ks are involved in signalling downstream of activated RTKs [39]. PI3K- 
Dluya Samuel Thagriki., Saudi J Biomed Res, Jan, 2022; 7(1): 25-32

C2 $\beta$ hence may mediate lipid signalling cascade in endocytic vesicles in feedback to receptor activation. $\mathrm{PI} 3 \mathrm{~K}-\mathrm{C} 2 \alpha$ and PI3K-C2 $\beta$ have been shown to interact with clathrin, an endocytic component. These interaction results in heightened lipid kinase activity as well as the production of $\mathrm{PI}_{3,4,5} \mathrm{P}_{3}$ in vitro $[36,38]$ signifying that $\mathrm{PI} 3 \mathrm{~K}-\mathrm{C} 2 \beta$ may convert $\mathrm{PI}_{4} \mathrm{P}$ and $\mathrm{PI}_{4,5} \mathrm{P}_{2}$ to $\mathrm{PI}_{3,4} \mathrm{P}_{2}$ and $\mathrm{PI}_{3,4,5} \mathrm{P}_{3}$ in vivo [40]. The ideal substrate for class II PI3Ks is PI in vitro. Das and colleagues demonstrated that INSTNs interact directly with new isoform of phosphoinositide 3-kinase (PI3K) through activation of PI3K-AKT pathway [4]. Precisely, INSTN-1 binding and activation of PI3K$\mathrm{C} 2 \beta$ is essential for neural cells survival during differentiation in vitro [4]. INSTN-1 knockdown in neural cells rises apoptosis and phenocopied by impediment of PI3K and AKT pathways and salvaged overexpression of PI3K-C2 $\beta$ or AKT. Decline survival of INSTN-1-knockdown in neuronal cells may not be attributed defective endocytosis because internalization of transferrin is not hampered in INSTN-1 knockdown cells. Proliferation of existing cells is unaltered by INSTN-1 knockdown implying that INSTN-1-PI3K pathway is necessary for only processing of differentiation [4]. Predescu and group also demonstrated that knockdown of INSTN-1 in human microvascular endothelial cells activates mitochondrial apoptosis pathway thru a yet undefined mechanism [5]. In a similar manner, PI3KC2 $\beta$ has been reported to have a canonical Ras binding domain (RBD) and co-localizes Ras and INSTN-1 on vesicles [41]. Nevertheless, Ras effectors PI3KC2 $\beta$ showed no interaction with activated Ras rather it is linked with inactive Ras. Characterization of the interaction showed nucleotidefree Ras bound to RBD of PI3KC2 $\beta$ and inhibition of lipid kinase activity in vitro. Furthermore, interaction of PI3KC2 $\beta$ with nucleotide-free Ras prohibited loading with nucleotide. Studies by Wong et al., also revealed that INSTN-1 interaction with PI3KC $2 \beta$ resulting in the dissociation of nucleotide-free Ras-PI3KC $2 \beta$ complex emanating in rapid GTP loading onto Ras leading to its activation. The above studies therefore support the hypothesis that INSTN-1 may serves as essential regulator of cell survival in many cell types.

\section{New insight into INSTNs role in viral endocytosis}

The role of INSTNs has been well reported in several diseases and regulating signalling pathways; however, few studies have highlighted the function of INSTNs in viral endocytosis. Recently, INSTNs were reported for mediating viral infectivity through various pathways such as AP-2, Cdc42 and N-WASP regulation. This review described recent insightful role of INSTNs in the regulation of viral endocytosis. Although INSTNs exhibits much of its functions in lower organisms, it is also implicated in viral endocytosis. Earlier in 2007, Lim and group showed that intersectin-2 interact with K15 protein of Kaposi's Sarcoma-Associated Herpesvirus by selective $\mathrm{SH} 3$ domain. They showed that PPLP motif binds to SH3-C domain of intersectin-2 in selective manner, K15 protein and intersectin- 2 colocalized distinct segment of the B-cells [42]. They proposed new role of K15 viral protein in intracellular trafficking. This was the first report on INSTN role during pathogenic endocytosis. Secondly, Dergai and group in 2013 showed INSTN-1 interaction with Epstein-Barr virus Latent Membrane Protein 2 (LMP2A). The interaction is mediated by SH3 domains of INSTN-1 at the N and C-tails of LMP2A. They found Shb adaptor and the Syk kinase as new INSTN-1 binding ligands. Their findings revealed INSTN-1 interaction with LMP2A occurred through Shb adaptor interacting with phosphorylated tyrosines of LMP2A and INSTN-1 SH3 domains. Their findings also showed that LMP2A affect other signalling pathways through INSTN-1 and Shb adaptors phosphorylation [43]. Thirdly, Snetkov and group in 2016 showed that direct interaction of NPF motifs at Cterminus of A36 with INSTN-1 and Epsin15 homology domains recruit AP-2 and clathrin mediate viral release and subsequent spreading as indicated in vaccinia virus [44]. Recently, Burbage and group in 2018 showed that mice deficient in INSTN-2, a G-nucleotide exchange factor (GEF) for Cdc42 interact with WASp and WIP exhibited high mortality rate, impaired antibody responses to vaccination, and reduced germinal centre formation during primary infection in Influenza A virus. $B$ cells deficient in INSTN-2 revealed decreased in CD84, SLAM and ICOSL expression that correlated with the reduction in forming conjugates with T-cells and differentiation into germinal centre cells. Their findings revealed the significance of INSTN-2 in regulating adaptive immune response during viral infectivity [45]. INSTN-2 is a multi-channel adaptor protein expressed as two INSTN-2S and INSTN-2L. isoforms. Both isoforms comprise of $\mathrm{SH} 3$ domains which support direct interactions with WASp and WIP and other multiple partners [46]. However, INSTN-2L has GEF domain containing DH (Dbl homology) domain attached to a PH (Pleckstrin homology) domain [13]. Remarkably, ITSN2-L GEF activity is selective for $\mathrm{Cdc} 42$ [47]. Figure 3 depict the interacting patterns with INSTN-1 and INSTN-2 during viral endocytosis. Furthermore, our recent findings from transcriptomic analysis of differentially expressed proteins of dengue virus depict INSTNs to be upregulated and utilised endocytic associated proteins and signalling pathways for their function (data not shown). We conclude that INSTNs are essential for viral endocytosis and as adaptive proteins that may mediate either viral entry, internalization or fusion in collaboration with other endocytic accessory proteins, hence the need for more study. 


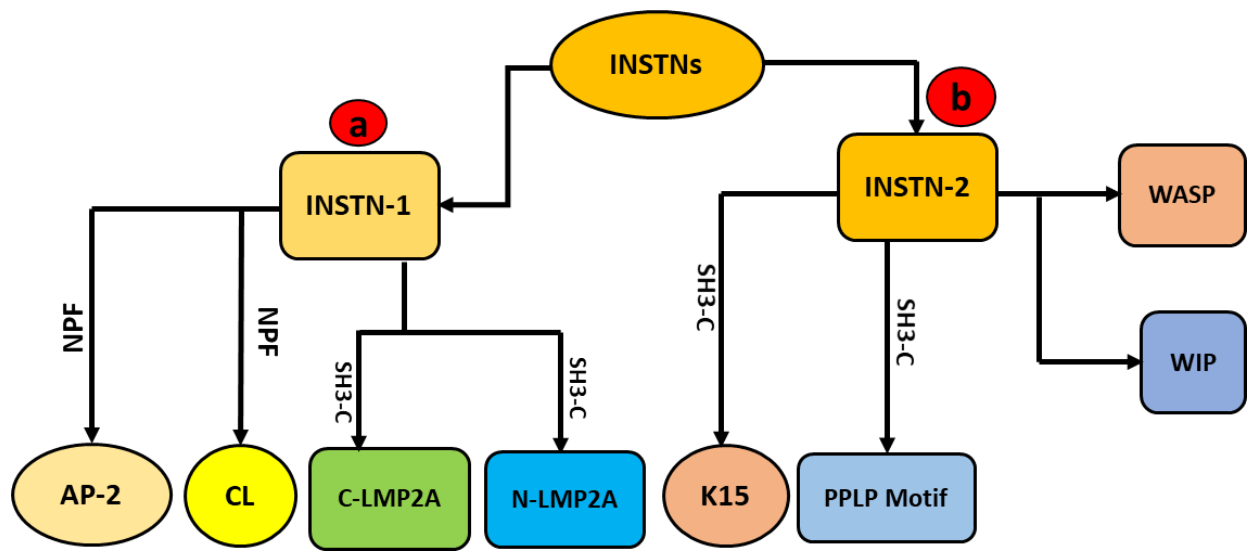

Figure 3: INSTNs interact with different viral proteins. (a) Direct interaction of NPF motifs at C-terminus of A36 with INSTN1 recruiting AP-2 and clathrin in vaccinia virus. INSTN-1 interaction is mediated by SH3 domains at N- and C-tails of Latent Membrane Protein 2 (LMP2A) in Epstein-Barr virus. (b) INSTN-2 interact with K15 protein of Kaposi's Sarcoma-Associated Herpesvirus by selective SH3 domain. PPLP motif binds to SH3-C domain of INSTN-2. INSTN-2 interact with WASp and WIP in Influenza A virus

\section{INSTN role in neurodegenerative diseases and Cancer}

INSTNs have been reported in neurodegenerative diseases commonly in Alzheimer Disease (AD), Down Syndrome (DS), Huntington diseases and cancer [48]. Briefly, INSTN-1 was overexpressed in patients reported with Down Syndrome. Human type INSTN-1 was found localized to $21 \mathrm{q} 22.2$ chromosome, a region closely related to phenotypic Down Syndrome. In vitro study from Drosophila showed that overexpression of the gene resulted in inhibition of endocytosis in neural and nonneural cells [49]. INSTN-1 was also reported as one of the most expressed genes in Alzheimer Disease [50, 51]. These diseases are characterized by early endosomal compartment [52]. In Huntington disease, elevated INSTN-1 lead to an increased accumulation of mutant hungtintin (htt) protein resulting to heightened neural dysfunction [53]. Russo and O'Bryan also demonstrated that INSTN-1 is required for neuroblastoma tumorigenesis. Silencing of INSTN-1 in vitro highly inhibited the independent growth of tumor cells and formation of tumor in xenograft assays independent of MYCN status. Their findings revealed that ITSN-1-PI3K-C2 $\beta$ pathway is important in neuroblastoma tumorigenesis and has specific role in neuroblastomas, a type of human cancer [54]. Their findings also showed that there may be additional INSTN-1 pathway for neuroblastoma tumorigenesis regulation since its function was not depicted as INSTN-1. Furthermore, Ma and colleagues also found that INSTN-1 is involved in migration and invasion of human glioma cells [55]. Their result revealed that INSTN-1 contribute to glioma cells movement and invasion through regulation of cytoskeletal formation, inducing adhesion as well as elevating the expression of MMP-9. The finding revealed INSTN-1 as a new target for therapeutic intervention in gliomas. Another study also revealed the essential role of INSTN-1 in the regulation on glioblastoma cells proliferation through the Raf/MEK/ERK pathway [56].

\section{CONCLUSION}

INSTNs been scaffold proteins has been shown to have multiple functions and regulate many biochemical pathways. INSTNs have earlier been well reported in neurodegenerative diseases. Recently they have been shown to be significance in viral endocytosis. So far, Herpesvirus, Epstein Barr virus, Vaccinia virus and Influenza A virus utilised INSTNs as endocytic adaptor proteins for multifunction purpose which may be responsible for viral entry, internalization or fusion. INSTNs function as endocytic adaptor, collaborate with other endocytic machineries and several signalling pathways for executing these tasks. Further investigation could provide new insight into other roles of INSTNs in enhancing viral infectivity through endocytosis, association with other endocytic proteins and the multifaceted signalling pathways.

\section{Acknowledgement}

CSIR and AcSIR is appreciated for academic support. TWAS-CSIR is acknowledged for $\mathrm{PhD}$ fellowship of Mr. Thagriki.

Funding: There is no separate funding support for this work.

\section{REFERENCES}

1. Predescu, S. A., Predescu, D. N., Timblin, B. K., Stan, R. V., \& Malik, A. B. (2003). Intersectin regulates fission and internalization of caveolae in endothelial cells. Molecular biology of the cell, 14(12), 4997-5010.

2. Hussain, N. K., Jenna, S., Glogauer, M., Quinn, C. C., Wasiak, S., Guipponi, M., ... \& McPherson, P. S. (2001). Endocytic protein intersectin-1 regulates actin assembly via Cdc42 and N-WASP. Nature cell biology, 3(10), 927-932.

3. Mohney, R. P., Das, M., Bivona, T. G., Hanes, R., Adams, A. G., Philips, M. R., \& O'Bryan, J. P. (2003). Intersectin activates Ras but stimulates transcription through an independent pathway 
Dluya Samuel Thagriki., Saudi J Biomed Res, Jan, 2022; 7(1): 25-32

involving JNK. Journal of Biological Chemistry, 278(47), 47038-47045.

4. Das, M., Scappini, E., Martin, N. P., Wong, K. A., Dunn, S., Chen, Y. J., ... \& O'Bryan, J. P. (2007). Regulation of neuron survival through an intersectin-phosphoinositide 3 '-kinase C2 $\beta$-AKT pathway. Molecular and cellular biology, 27(22), 7906-7917.

5. Predescu, S. A., Predescu, D. N., Knezevic, I., Klein, I. K., \& Malik, A. B. (2007). Intersectin-1s regulates the mitochondrial apoptotic pathway in endothelial cells. Journal of biological chemistry, 282(23), 17166-17178.

6. Sparks, A. B., Hoffman, N. G., McConnell, S. J., Fowlkes, D. M., \& Kay, B. K. (1996). Cloning of ligand targets: systematic isolation of $\mathrm{SH} 3$ domaincontaining proteins. Nature biotechnology, 14(6), 741-744.

7. Yamabhai, M., Hoffman, N. G., Hardison, N. L., McPherson, P. S., Castagnoli, L., Cesareni, G., \& Kay, B. K. (1998). Intersectin, a novel adaptor protein with two Eps15 homology and five Src homology 3 domains. Journal of Biological Chemistry, 273(47), 31401-31407.

8. Yu, Y., Chu, P. Y., Bowser, D. N., Keating, D. J., Dubach, D., Harper, I., ... \& Pritchard, M. A. (2008). Mice deficient for the chromosome 21 ortholog Itsn1 exhibit vesicle-trafficking abnormalities. Human molecular genetics, 17(21), 3281-3290.

9. Ma, Y. J., Okamoto, M., Gu, F., Obata, K., Matsuyama, T., Desaki, J., ... \& Sakanaka, M. (2003). Neuronal distribution of EHSH1/intersectin: Molecular linker between clathrin-mediated endocytosis and signaling pathways. Journal of neuroscience research, 71(4), 468-477.

10. Guipponi, M., Scott, H. S., Chen, H., Schebesta, A., Rossier, C., \& Antonarakis, S. E. (1998). Two isoforms of a human intersectin (ITSN) protein are produced by brain-specific alternative splicing in a stop codon. Genomics, 53(3), 369-376.

11. Pucharcos, C., Casas, C., Nadal, M., Estivill, X., \& de la Luna, S. (2001). The human intersectin genes and their spliced variants are differentially expressed. Biochimica et Biophysica Acta (BBA)Gene Structure and Expression, 1521(1-3), 1-11.

12. Sengar, A. S., Wang, W., Bishay, J., Cohen, S., \& Egan, S. E. (1999). The EH and SH3 domain Ese proteins regulate endocytosis by linking to dynamin and Eps15. The EMBO journal, 18(5), 1159-1171.

13. Pucharcos, C., Estivill, X., \& de la Luna, S. (2000). Intersectin 2, a new multimodular protein involved in clathrin-mediated endocytosis. FEBS letters, 478(1-2), 43-51.

14. Santolini, E., Salcini, A. E., Kay, B. K., Yamabhai, M., \& Di Fiore, P. P. (1999). The EH network. Experimental cell research, 253(1), 186209.
15. Fernández-Chacón, R., Achiriloaie, M., Janz, R., Albanesi, J. P., \& Südhof, T. C. (2000). SCAMP1 function in endocytosis. Journal of Biological Chemistry, 275(17), 12752-12756.

16. Martina, J. A., Bonangelino, C. J., Aguilar, R. C., \& Bonifacino, J. S. (2001). Stonin 2An Adaptorlike Protein That Interacts with Components of the Endocytic Machinery. The Journal of cell biology, 153(5), 1111-1120.

17. Adams, A., Thorn, J. M., Yamabhai, M., Kay, B. K., \& O'Bryan, J. P. (2000). Intersectin, an adaptor protein involved in clathrin-mediated endocytosis, activates mitogenic signaling pathways. Journal of Biological Chemistry, 275(35), 27414-27420.

18. Pechstein, A., Bacetic, J., Vahedi-Faridi, A., Gromova, K., Sundborger, A., Tomlin, N., ... \& Haucke, V. (2010). Regulation of synaptic vesicle recycling by complex formation between intersectin 1 and the clathrin adaptor complex AP2. Proceedings of the National Academy of Sciences, 107(9), 4206-4211.

19. Gubar, O., Morderer, D., Tsyba, L., Croisé, P., Houy, S., Ory, S., ... \& Rynditch, A. (2013). Intersectin: the crossroad between vesicle exocytosis and endocytosis. Frontiers in endocrinology, 4, 109.

20. Hussain, N. K., Yamabhai, M., Ramjaun, A. R., Guy, A. M., Baranes, D., O'Bryan, J. P., ... \& McPherson, P. S. (1999). Splice variants of intersectin are components of the endocytic machinery in neurons and nonneuronal cells. Journal of Biological Chemistry, 274(22), 15671-15677.

21. O’Bryan, J. P. (2010). Intersecting pathways in cell biology. Science signaling, 3(152), re10-re10.

22. Henne, W. M., Boucrot, E., Meinecke, M., Evergren, E., Vallis, Y., Mittal, R., \& McMahon, H. T. (2010). FCHo proteins are nucleators of clathrin-mediated endocytosis. Science, 328(5983), 1281-1284.

23. Tong, X. K., Hussain, N. K., Adams, A. G., O'Bryan, J. P., \& McPherson, P. S. (2000). Intersectin can regulate the Ras/MAP kinase pathway independent of its role in endocytosis. Journal of Biological Chemistry, 275(38), 29894-29899.

24. Tong, X. K., Hussain, N. K., De Heuvel, E., Kurakin, A., Abi-Jaoude, E., Quinn, C. C., ... \& McPherson, P. S. (2000). The endocytic protein intersectin is a major binding partner for the Ras exchange factor mSos1 in rat brain. The EMBO journal, 19(6), 1263-1271.

25. Martin, N. P., Mohney, R. P., Dunn, S., Das, M., Scappini, E., \& O'Bryan, J. P. (2006). Intersectin regulates epidermal growth factor receptor endocytosis, ubiquitylation, and signaling. Molecular pharmacology, 70(5), $1643-$ 1653.

26. Okur, M. N., Russo, A., \& O'Bryan, J. P. (2014). Receptor tyrosine kinase ubiquitylation involves 
Dluya Samuel Thagriki., Saudi J Biomed Res, Jan, 2022; 7(1): 25-32

the dynamic regulation of Cbl-Spry2 by intersectin 1 and the Shp2 tyrosine phosphatase. Molecular and cellular biology, 34(2), 271-279.

27. Vieira, A. V., Lamaze, C., \& Schmid, S. L. (1996). Control of EGF receptor signaling by clathrinmediated endocytosis. Science, 274(5295), 20862089.

28. Mor, A., \& Philips, M. R. (2006). Compartmentalized ras/mapk signaling. Annu. Rev. Immunol., 24, 771-800.

29. Lu, A., Tebar, F., Alvarez-Moya, B., López-Alcalá, C., Calvo, M., Enrich, C., ... \& Bachs, O. (2009). A clathrin-dependent pathway leads to KRas signaling on late endosomes en route to lysosomes. Journal of Cell Biology, 184(6), 863879.

30. Nimnual, A. S., Yatsula, B. A., \& Bar-Sagi, D. (1998). Coupling of Ras and Rac guanosine triphosphatases through the Ras exchanger Sos. Science, 279(5350), 560-563.

31. Jenna, S., Hussain, N. K., Danek, E. I., Triki, I., Wasiak, S., McPherson, P. S., \& Lamarche-Vane, N. (2002). The activity of the GTPase-activating protein CdGAP is regulated by the endocytic protein intersectin. Journal of Biological Chemistry, 277(8), 6366-6373.

32. O'Bryan, J. P., Mohney, R. P., \& Oldham, C. E. (2001). Mitogenesis and endocytosis: What's at the INTERSECTIoN?. Oncogene, 20(44), 6300-6308.

33. Nishimura, T., Yamaguchi, T., Tokunaga, A., Hara, A., Hamaguchi, T., Kato, K., ... \& Kaibuchi, K. (2006). Role of numb in dendritic spine development with a $\mathrm{Cdc} 42 \mathrm{GEF}$ intersectin and EphB2. Molecular biology of the cell, 17(3), 12731285.

34. Irie, F., \& Yamaguchi, Y. (2002). EphB receptors regulate dendritic spine development via intersectin, $\mathrm{Cdc} 42$ and N-WASP. Nature neuroscience, 5(11), 1117-1118.

35. Schmidt, M. H., \& Dikic, I. (2005). The Cbl interactome and its functions. Nature reviews Molecular cell biology, 6(12), 907-919.

36. Gaidarov, I., Smith, M. E., Domin, J., \& Keen, J. H. (2001). The class II phosphoinositide 3-kinase $\mathrm{C} 2 \alpha$ is activated by clathrin and regulates clathrinmediated membrane trafficking. Molecular cell, 7(2), 443-449.

37. Domin, J., Gaidarov, I., Smith, M. E., Keen, J. H., \& Waterfield, M. D. (2000). The class II phosphoinositide 3-kinase $\mathrm{PI} 3 \mathrm{~K}-\mathrm{C} 2 \alpha$ is concentrated in the trans-Golgi network and present in clathrin-coated vesicles. Journal of Biological Chemistry, 275(16), 11943-11950.

38. Wheeler, M., \& Domin, J. (2006). The N-terminus of phosphoinositide 3-kinase-C2 $\beta$ regulates lipid kinase activity and binding to clathrin. Journal of cellular physiology, 206(3), 586-593.

39. Wheeler, M., \& Domin, J. (2001). Recruitment of the class II phosphoinositide 3-kinase C2 $\beta$ to the epidermal growth factor receptor: role of
Grb2. Molecular and cellular biology,21(19), 6660-6667.

40. Traer, C. J., Foster, F. M., Abraham, S. M., \& Fry, M. J. (2006). Are class II phosphoinositide 3kinases potential targets for anticancer therapies?. Bulletin du cancer, 93(5), 10053-10058.

41. Wong, K. A., Russo, A., Wang, X., Chen, Y. J., Lavie, A., \& O'Bryan, J. P. (2012). A new dimension to Ras function: A novel role for nucleotide-free Ras in class II phosphatidylinositol 3-kinase beta (PI3KC2 $\beta$ ) regulation. PLOS ONE, 7(9), e45360.

42. Lim, C. S., Seet, B. T., Ingham, R. J., Gish, G., Matskova, L., Winberg, G., ... \& Pawson, T. (2007). The K15 protein of Kaposi's sarcomaassociated herpesvirus recruits the endocytic regulator intersectin 2 through a selective $\mathrm{SH} 3$ domain interaction. Biochemistry, 46(35), 9874 9885.

43. Dergai, O., Dergai, M., Skrypkina, I., Matskova, L., Tsyba, L., Gudkova, D., \& Rynditch, A. (2013). The LMP2A protein of Epstein-Barr virus regulates phosphorylation of ITSN1 and Shb adaptors by tyrosine kinases. Cellular signalling, 25(1), 33-40.

44. Snetkov, X., Weisswange, I., Pfanzelter, J., Humphries, A. C., \& Way, M. (2016). NPF motifs in the vaccinia virus protein A36 recruit intersectin1 to promote Cdc42: N-WASP-mediated viral release from infected cells. Nature Microbiology, 1(10), 1-11.

45. Burbage, M., Gasparrini, F., Aggarwal, S., Gaya, M., Arnold, J., Nair, U., ... \& Batista, F. D. (2018). Tuning of in vivo cognate BT cell interactions by Intersectin 2 is required for effective anti-viral B cell immunity. Elife, 7, e26556.

46. Gryaznova, T., Kropyvko, S., Burdyniuk, M., Gubar, O., Kryklyva, V., Tsyba, L., \& Rynditch, A. (2015). Intersectin adaptor proteins are associated with actin-regulating protein WIP in invadopodia. Cellular signalling, 27(7), 1499 1508.

47. Novokhatska, O., Dergai, M., Houssin, N., Tsyba, L., Moreau, J., \& Rynditch, A. (2011). Intersectin 2 nucleotide exchange factor regulates Cdc42 activity during Xenopus early development. Biochemical and biophysical research communications, 408(4), 663-668.

48. Hunter, M. P., Russo, A., \& O'Bryan, J. P. (2013). Emerging roles for intersectin (ITSN) in regulating signaling and disease pathways. International journal of molecular sciences, 14(4), 7829-7852.

49. Keating, D. J., Chen, C., \& Pritchard, M. A. (2006). Alzheimer's disease and endocytic dysfunction: clues from the Down syndromerelated proteins, DSCR1 and ITSN1. Ageing research reviews, 5(4), 388-401.

50. Wilmot, B., McWeeney, S. K., Nixon, R. R., Montine, T. J., Laut, J., Harrington, C. A., ... \& Kramer, P. L. (2008). Translational gene mapping 
of cognitive decline. Neurobiology of aging, 29(4), 524-541.

51. Dunckley, T., Beach, T. G., Ramsey, K. E., Grover, A., Mastroeni, D., Walker, D. G., ... \& Stephan, D. A. (2006). Gene expression correlates of neurofibrillary tangles in Alzheimer's disease. Neurobiology of aging, 27(10), 1359-1371.

52. Nixon, R. A. (2005). Endosome function and dysfunction in Alzheimer's disease and other neurodegenerative diseases. Neurobiology of aging, 26(3), 373-382.

53. Scappini, E., Koh, T. W., Martin, N. P., \& O'Bryan, J. P. (2007). Intersectin enhances huntingtin aggregation and neurodegeneration through activation of c-Jun-NH2-terminal kinase. Human molecular genetics, 16(15), 1862-1871.

54. Russo, A., \& O'Bryan, J. P. (2012). Intersectin 1 is required for neuroblastoma tumorigenesis. Oncogene, 31(46), 4828-4834.

55. Ma, Y., Wang, B., Li, W., Liu, X., Wang, J., Ding, T., ... \& Gu, F. (2011). Intersectin1-s is involved in migration and invasion of human glioma cells. Journal of neuroscience research,89(7), 1079-1090.

56. Gu, F., Zhang, H., Qin, F., Liu, X., Li, W., Fu, L., ... \& Ma, Y. (2015). Intersectin1-s, A multidomain adapter protein, Is essential for malignant glioma proliferation. Glia, 63(9), 1595-1605. 